\title{
Automated Planning of Scan Geometries in Spine MRI Scans
}

\author{
Vladimir Pekar ${ }^{1, \star}$, Daniel Bystrov ${ }^{1}$, Harald S. Heese ${ }^{1}$, Sebastian P. M. Dries ${ }^{1}$, \\ Stefan Schmidt ${ }^{1,2}$, Rüdiger Grewer ${ }^{1}$, Chiel J. den Harder ${ }^{3}$, \\ René C. Bergmans ${ }^{3}$, Arjan W. Simonetti ${ }^{3}$, and Arianne M. van Muiswinkel ${ }^{3}$ \\ ${ }^{1}$ Philips Research Europe - Hamburg, Germany \\ ${ }^{2}$ University of Mannheim, Germany \\ ${ }^{3}$ Philips Medical Systems, Best, The Netherlands \\ vladimir . pekar@philips.com
}

\begin{abstract}
Consistency of MR scan planning is very important for diagnosis, especially in multi-site trials and follow-up studies, where disease progress or response to treatment is evaluated. Accurate manual scan planning is tedious and requires skillful operators. On the other hand, automated scan planning is difficult due to relatively low quality of survey images ("scouts") and strict processing time constraints. This paper presents a novel method for automated planning of MRI scans of the spine. Lumbar and cervical examinations are considered, although the proposed method is extendible to other types of spine examinations, such as thoracic or total spine imaging. The automated scan planning (ASP) system consists of an anatomy recognition part, which is able to automatically detect and label the spine anatomy in the scout scan, and a planning part, which performs scan geometry planning based on recognized anatomical landmarks. A validation study demonstrates the robustness of the proposed method and its feasibility for clinical use.
\end{abstract}

\section{Introduction}

The diagnostic value of MRI scans greatly depends on the accuracy and consistency of scan planning. This procedure is typically carried out on a low resolution (often just a few orthogonal slices) survey dataset, the so-called "scout", in which scan geometries, such as off-center, angulation and field-of-view, are determined manually by the operator. The operators often need to plan several diagnostic scans with different parameters during one session, which requires high concentration and is potentially error-prone. Every operator has an individual planning style depending on his/her level of experience, training, etc. All these factors negatively influence the consistency and reproducibility in the resulting diagnostic scan geometries.

In order to improve the MR acquisition workflow, several research groups have proposed automated scan planning (ASP) methods for different anatomies, e.g. neurocranial [12], cardiac [34], and knee [5].

\footnotetext{
^ V. Pekar is currently with Philips Medical Systems, Markham, ON, Canada.
} 
Spine examinations represent one of the most important clinical applications of MRI. However, automated spine planning is a particularly difficult task, since several peculiarities have to be taken into consideration: i) Spine has a repetitive anatomical structure, so the correct order of visible vertebrae needs to be recognized and labeled. ii) The number of vertebrae may vary from one individual to another. iii) The appearance of vertebrae and intervertebral discs may be affected by different pathologies, e.g. deformity (scoliosis), fracture, neoplasm, degeneration, etc. iv) The ASP system has to be able to deal with partial spine acquisitions, for example, in examinations of the lower (lumbar) or upper (cervical) spine. The exact number of visible vertebrae in such examinations is not known in advance and the use of global registration or structural model-based methods for anatomy detection is difficult.

Robust anatomy recognition is a core requirement for an ASP system to be advantageous over manual planning in clinical routine. Automated spine detection and labeling is a technically challenging task even in diagnostic quality MRI. Peng et al. 6] aim at detecting the intervertebral discs in adjacent 2-D sagittal slices for segmentation of the whole spine column. A feature detector based on template matching is applied to detect candidates for disc centers followed by local post-processing. A semi-automated approach for labeling intervertebral discs in whole spine has been proposed by Weiss et al. 7]. After intensity correction step, the operator provides a seed point in the $\mathrm{C} 2-\mathrm{C} 3$ disc to start the search procedure based on intensity thresholds. Vrtovec et al. 8] detect the spine by searching for circular areas of homogeneous intensity in axial slices. The performance of the above mentioned approaches strongly depends on good image quality with reproducible contrasts. This may not be the case for scout data, of which the quality is usually limited by the clinically acceptable scanning time.

This paper develops a new approach for fast and robust automated spine detection and labeling in 3-D scout images. Analogously to earlier work [25], the detected anatomical landmarks are used to learn pre-defined geometries from a training set provided by the operator. In this way, the ASP system allows to consistently plan scan geometries tailored to individual needs and preferences of the radiologist. The focus of this paper is on planning lumbar and cervical partial examinations, although the approach can be extended to other types of examinations, e.g. thoracic or total spine.

The reliability of the proposed method is demonstrated by validating the anatomy detection on 60 spine scout images (30 cervical and 30 lumbar datasets). The experimental part of the paper also illustrates automated learning of scan plan geometries and their transfer from one area of the spine to another.

\section{Method}

Automated scan planning starts with the acquisition of a 3-D low-resolution scout scan. The scout image is analyzed by the anatomy recognition algorithm, which automatically detects the intervertebral discs and labels the corresponding vertebrae. In a clinical environment, the system would typically ask the operator 
to confirm (and, if needed, even adapt) the correctness of anatomy detection. After the labeling step, the positions and orientations of the intervertebral discs are used to generate a set of landmarks required for automated geometry planning.

\subsection{Image Data}

Scout protocols generally require a compromise between obtaining the image quality and resolution sufficient to successfully cope with the task of scan planning, and limited acquisition time, which should be kept as short as possible. The spine planning method in this work uses dedicated 3-D T1-weighted fast field echo (FFE) scans having sagittal slice orientation with 400 x 400 x $270 \mathrm{~mm}$ field of view $(1.25 \times 1.25 \times 1.5 \mathrm{~mm}$ voxel size) and acquisition time of $40 \mathrm{~s}$. The datasets were acquired on a $1.5 \mathrm{~T}$ Philips Achieva MR scanner.

\subsection{Anatomy Recognition}

The spine detection is implemented in the form of sequential application of three processing steps aiming at fast and reliable detection of the intervertebral discs.

Detection of disc candidates. As a pre-processing step, an interest point detector is applied to simplify the spine detection problem through analyzing a relatively small number of candidates. Since intervertebral discs appear as bright line-like structures in sagittal or coronal slices, our interest point detector is based on a special filter which detects approximately horizontal lines in sagittal 2-D slices of the scout image. This approach is computationally more efficient than searching for disc-like structures in 3-D. The filter response used is based on eigenanalysis of the image Hessian and is defined for image position $\mathbf{x}$ as: $V(\mathbf{x}, s)=\exp \left\{-\frac{\lambda_{1}{ }^{2} / \lambda_{2}{ }^{2}}{a^{2}}\right\}\left(1-\exp \left\{-\frac{\lambda_{1}{ }^{2}+\lambda_{2}{ }^{2}}{b^{2}}\right\}\right)$, where $\lambda_{1}$ and $\lambda_{2}$ are the eigenvalues of the Hessian matrix at scale $s$ with $\left|\lambda_{1}\right| \leq\left|\lambda_{2}\right|$, and $a$ and $b$ are sensitivity weights [9]. The value of $a$ was set to 500 in this work, and the value of $b$ was set to half of the maximum Hessian norm, as proposed in [9]. The first term suppresses blob-like structures while the second term suppresses the influence of the background noise. Admissible intensity range and principal Hessian eigenvector orientation are used to improve selectivity of the filter.

The filter is applied on a isotropically downsampled image with the voxel size of $3 \mathrm{~mm}^{3}$, using the scale parameter $s=1$. The filtered dataset is next converted to a binary image by applying the threshold $V>10^{-4}$ and cleaned by morphological opening using a rectangular structural element. The centers of mass of the 3-D connected components in the filtered image are considered as candidates for the intervertebral disc centers. Additionally, the smallest principal component of each 3-D segment is computed to estimate the orientation vector orthogonal to the disc.

Spine detection. Unambiguous discrimination between true disc centers and false ones in the set of candidates is a very difficult task, even by exploiting the structural connectivity and vertical orientation of the spine column. We follow 
a different strategy and aim at reliably finding one single point belonging to the spine. Starting from that point, a progressive search is initiated which extracts the whole visible spine using prior structural knowledge.

Since the spine column can be considered as a tubular structure, most of the false candidates can be removed by applying an iterative procedure which removes candidates that form point triplets with angles in the range $[\pi / 4,3 \pi / 4]$. This is done to eliminate segments with unrealistically high curvature. In each pass, the candidate point responsible for the largest number of inadmissible triplets is eliminated from the set and the procedure is repeated until no such points are left. The topmost candidate point from the remaining set is marked as the first point belonging to the spine column in lumbar scouts. Analogously, the bottommost candidate point is used for cervical scouts.

To improve the performance and robustness of the above method, a subset of 15 candidates mostly resembling intervertebral

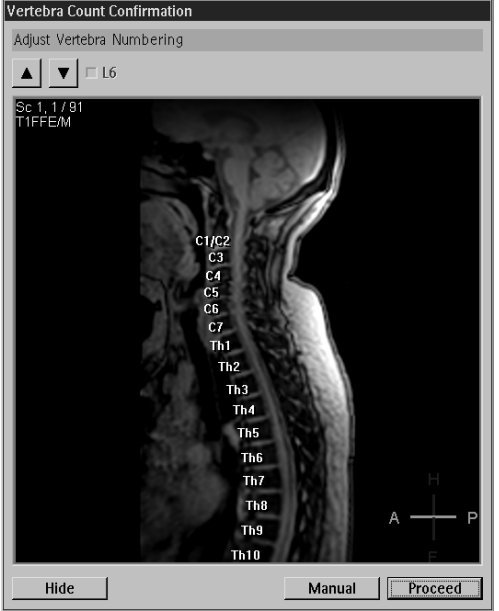

Fig. 1. GUI fragment with automatically labeled cervical scout discs is taken instead of the whole set. This reflects the maximum number of visible discs in partial spine examinations. In order to select these candidates, the corresponding location in the image is evaluated for similarity with an intervertebral disc template represented by a triangulated surface mesh and positioned according to the disc orientation vector computed in the previous step. The similarity value for the $k$-th candidate is defined as: $S_{k}=\frac{1}{N} \sum_{i=1}^{N} \mathbf{n}_{i}^{T} \mathbf{g}_{i, k}$, where $N$ is the number of triangles in the disc template mesh, $\mathbf{n}$ is the triangle normal unit vector and $\mathbf{g}$ is the normalized image gradient, computed at the triangle position.

Spine labeling. Starting at the first spine point, a progressive search is carried out upwards/downwards aiming at extracting the disc centers in the visible spine column from all candidates detected as interest points. The next spine point is selected as lying at a certain distance from the previously detected one, where the orientation of the corresponding intervertebral disc is used to define the search direction. If a candidate is missing at the expected position, e.g. due to pathology or image artifacts, an artificial disc candidate is inserted and searching continues using the same direction.

The search is terminated when several consecutive missing candidates are encountered. This criterion reliably identifies the $\mathrm{C} 2-\mathrm{C} 3$ disc in the cervical spine owing to the characteristic form of the $\mathrm{C} 2$ vertebra. In the lumbar spine, the L5$\mathrm{S} 1$ disc can be found as being the one with the maximum forward angulation about the axis in right-left direction of the patient. After the entire set of visible discs are extracted, the vertebrae are labeled w.r.t. the disc found last, see Fig. 1. 


\subsection{Scan Geometry Planning}

Analogously to previous work [2]5], the method uses a training set of manually planned geometries to propose a planning based on correlation with the automatically detected anatomical landmarks. On the training samples, rigid registration of the automatically detected landmarks is carried out. A robust multi-variate median approach [10] has to be employed in the computation of the "atlas" landmarks, since anatomical variability as well as variability in the landmark extraction impede perfect alignment. The variance of the landmark positions in the training samples w.r.t. the position of the corresponding "atlas" landmark furthermore determines its contribution to the proposed planning. Finally, the planning algorithm performs rigid registration of the detected set of landmarks from the current image with the "atlas" landmarks from the training set based on these contributions.

Considerable contrast fluctuations in the scout protocol and possible presence of severe pathologies, e.g. fractures, make accurate segmentation of structures such as vertebrae or intervertebral discs for the purpose of landmark extraction difficult. Instead, positions and orientations of the detected intervertebral discs are used to define fixed extents around each disc, whose corner points are used as landmarks. To account for the specific repetitive character of the spine anatomy, the planning concept has been extended to transfer plans from one particular area into another. For example, if the training set consists of lumbar anatomy plans only, the system is still capable of planning on cervical scouts.

\section{Results}

Validation of the approach has been carried out using two criteria: i) robustness of anatomy detection and ii) quality of automatically generated plans.

Validation of anatomy detection. Two cervical and two lumbar scout images of 15 different volunteers were acquired. The volunteers were asked to change their pose in-between, while the global positioning of each subject was kept fairly consistent. Ground-truth information regarding the position of the visible intervertebral discs was generated for all 60 datasets by a clinical expert.

In a first step, the performance of the disc candidate detection was evaluated, showing a sensitivity of $95.6 \%$ for the interest point detector. As the interest point detector responds on all horizontal line-like structures in the image, the number of false disc candidate detections is rather high, especially in cervical scouts owing to the smaller size of the intervertebral discs. The detailed statistics discriminating between cervical and lumbar scouts are summarized in Table 1

Secondly, the spine detection was evaluated. Fig. 2 illustrates the effect of dramatically reducing the number of false candidates arising in disc candidate detection by applying the spine detection procedure presented in Section 2.2 . The final candidate that was selected at the end of the procedure corresponded in all 60 images to a valid detection of an intervertebral disc. In 28 out of 30 lumbar images, the detected point corresponded to the uppermost candidate, in 
Table 1. Sensitivity results for detection of disc candidates in 60 datasets

\begin{tabular}{|l|l|l|l|}
\hline & cervical & lumbar & all \\
\hline visible discs (ground truth) & 387 & 289 & 676 \\
\hline overall candidates & 4480 & 841 & 5321 \\
\hline false candidates & 4111 & 564 & 4675 \\
\hline true detections & $369(95.3 \%)$ & $277(95.8 \%)$ & $646(95.6 \%)$ \\
\hline failed detections & $18(4.7 \%)$ & $12(4.2 \%)$ & $30(4.4 \%)$ \\
\hline
\end{tabular}

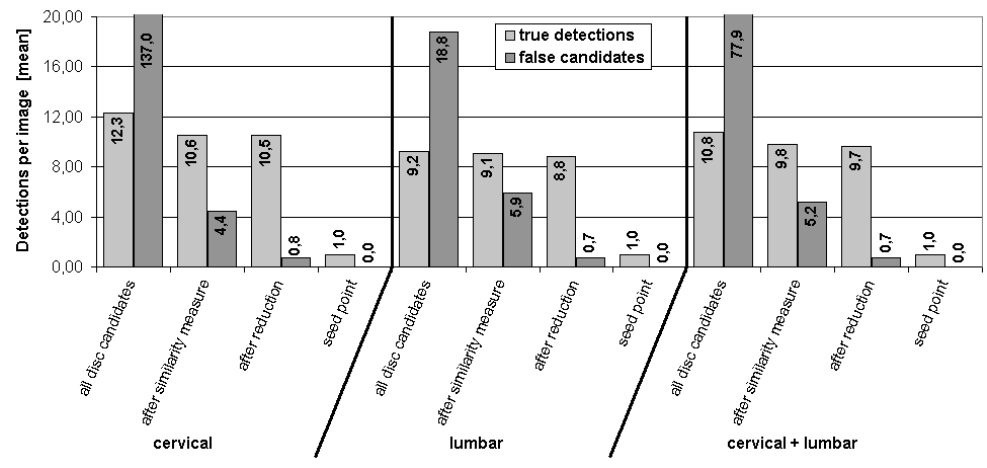

Fig. 2. Comparison of mean values per image for true detections and false candidates after different steps in the spine detection procedure

two images it was shifted downwards by one. Similarly, in 27 out of 30 cervical images the detected point corresponded to the lowermost candidate, in three images it was shifted upwards by one. These results imply that by performing the propagation process for the spine labeling, none of the intervertebral discs, that are within the clinically relevant area, are prone to be missed due to bad initialization.

Finally, the results of spine labeling were analyzed. In all 30 cervical images, the labeling was correct with the exception of one dataset, where one extra disc was erroneously detected. In particular, the progressive search was able to recover all relevant intervertebral discs for which disc candidate detection failed. Correspondingly, the labeling was correct in 25 out of 30 lumbar scouts. In the remaining 5 images L4-L5 was erroneously detected as the lowermost disc, however, in a clinical environment this error can be corrected by the operator.

Validation of automated planning. Qualitative assessment of consistency of the presented method with manual planning was carried out using 12 survey datasets. For the cervical and lumbar regions, manual plan sets were defined in six images each. Every plan set consisted of a mid-sagittal view of the total cervical or lumbar spine and two transverse views at different segmental levels: lower third of the vertebral body C6 or L4 and the disc below. Five plans were 

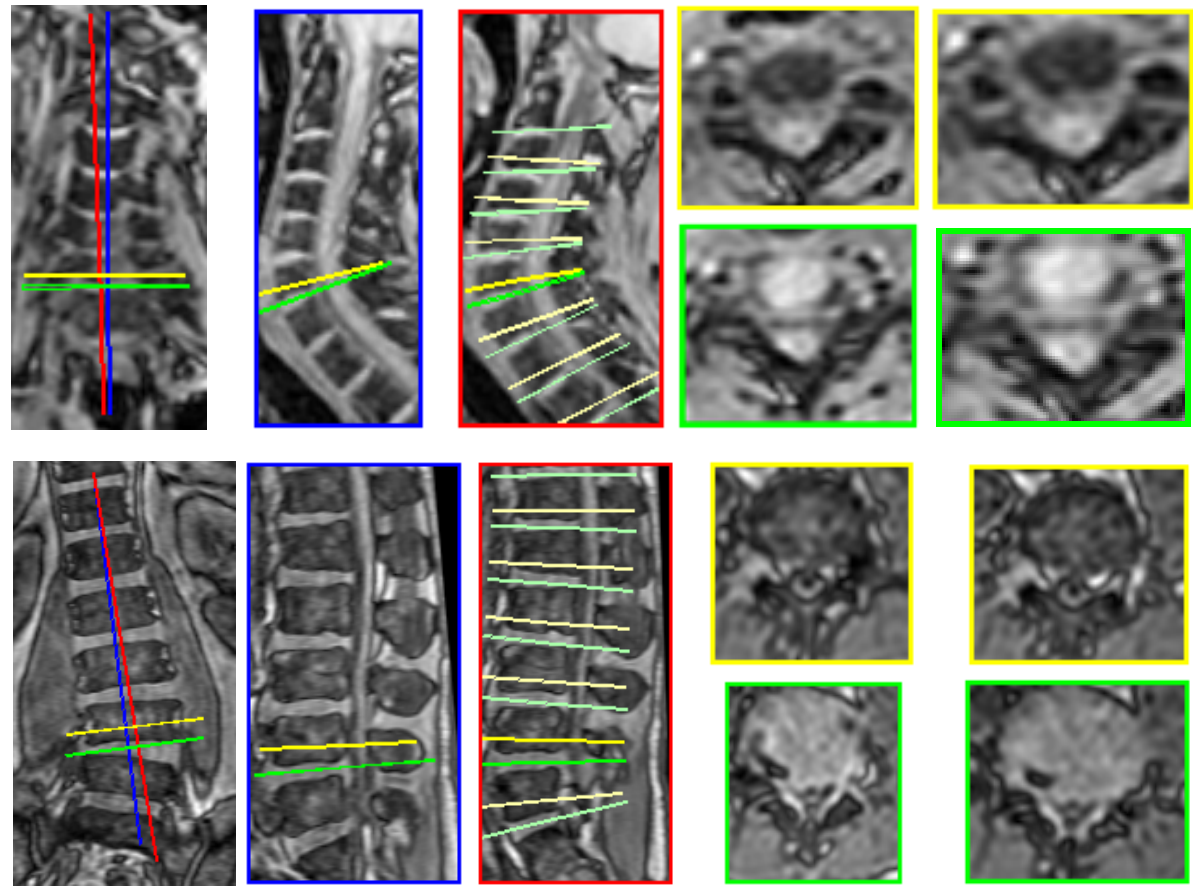

Fig. 3. Upper row: cervical, lower row: lumbar; left to right: manually planned frontal view, manually planned midsagittal view, automatically planned midsagittal view, manually planned vertebra/disc, automatically planned vertebra/disc. The blue and the red line in the frontal view correspond to the manually and automatically planned midsagittal planes. The yellow lines correspond to the planned vertebrae, the green lines correspond to the discs, the resulting transversal views are shown at the right.

based on the images of healthy volunteers from the anatomy detection validation study. These plans were used to train the ASP algorithm. For the sixth dataset, displaying a pathology, the corresponding plans were generated automatically and compared with the manual plans. The whole process including automated anatomy recognition and planning took about 6 seconds on a $2.4 \mathrm{GHz}$ Linux PC.

The results were inspected visually as shown in Fig. 3, where the additional frontal view facilitates comparison of the automated and manual plans. The results reveal consistent planning of the requested segments. Furthermore, the 3rd column from the left in Fig. 3 shows the algorithm's successful extrapolation of the transverse plans to other segments.

A small in-plane rotation between the manually and automatically planned mid-sagittal views can be observed. Nevertheless, the automatically planned views have been rated as fully acceptable by a clinical expert. Note that the slight lumbar scoliosis and apparent cervical hyperlordosis were not present in the training data, which emphasizes the robustness of the method. 


\section{Conclusion}

This paper has presented a novel method for automatically planning scan geometries in spine MRI scans. The approach makes use of automatically detected anatomical landmarks to learn and plan scan geometries for lumbar and cervical partial spine examinations. Since the number of visible vertebrae in such examinations is not known in advance, the proposed anatomy detection approach is based on analyzing locally extracted features aimed at determining the positions and orientations of intervertebral discs. The results of the validation study showed the anatomy labeling being correct in 54 out of 60 test datasets and shifted one level in the remaining 6 images. Based on a small number of training examples, automated planning showed consistent results for a clinical dataset with pathology, and in transferring plans to the areas of the spine other than those used for training. With the overall processing time of about 6 seconds, the proposed method is feasible for clinical use.

\section{References}

1. Itti, L., Chang, L., Ernst, T.: Automatic scan prescription for brain MRI. Magnetic Resonance in Medicine 45(3), 486-494 (2001)

2. Young, S., Bystrov, D., Netsch, T., Bergmans, R., van Muiswinkel, A., Visser, F., Springorum, R., Gieseke, J.: Automated planning of MRI neuro scans. In: Proc. of SPIE Medical Imaging, San Diego, CA, USA pp. 61441M-1-61441M-8 (2006)

3. Lelieveldt, R.P.F., van der Geest, R.J., Lamb, H.J., Kayser, H.W.M., Reiber, J.H.C.: Automated observer-independent acquisition of cardiac short-axis MR images: A pilot study. Radilogy 221, 537-542 (2001)

4. Jackson, C.E., Robson, M.D., Francis, J.M., Noble, J.A.: Computerized planning of the acquisition of cardiac MR images. Computerized Imaging and Graphics 28(7), 411-418 (2004)

5. Bystrov, D., Pekar, V., Young, S., Dries, S.P.M., Heese, H.S., van Muiswinkel, A.M.: Automated planning of MRI scans of knee joints. In: Proc. of SPIE Medical Imaging, San Diego, CA, USA pp. 65902Z-1-65902Z-9 (2007)

6. Peng, Z., Zhong, J., Wee, W., Lee, J.H.: Automated vertebra detection and segmentation from the whole spine MR images. In: Proc. of Engineering in Medicine and Biology, IEEE-EMBS, Shanghai, China, pp. 2527-2530 (2005)

7. Weiss, K.L., Storrs, J.M., Banto, R.B.: Automated spine survey iterative scan technique. Radiology 239(1), 255-262 (2006)

8. Vrtovec, T., Ourselin, S., Gomes, L., Likar, B., Pernus, F.: Generation of curved planar reformations from magnetic resonance images of the spine. In: Larsen, R., Nielsen, M., Sporring, J. (eds.) MICCAI 2006. LNCS, vol. 4191, pp. 135-143. Springer, Heidelberg (2006)

9. Frangi, A., Niessen, W., Hoogeveen, R., van Walsum, T., Viergever, M.: Multiscale vessel enhancement filtering. In: Wells, W., et al. (eds.) Proc. of MICCAI 1998, Cambridge, MA, USA, pp. 130-137 (1998)

10. Vardi, Y., Zhang, C.H.: The multivariate 11-median and associated data depth. Proc. National Academy of Sciences USA 97(4), 1423-1436 (2000) 